Check for updates

Cite this: RSC Adv., 2017, 7, 20494

Received 5th January 2017 Accepted 27th March 2017

DOI: $10.1039 / \mathrm{c} 7 \mathrm{ra00184c}$

rsc.li/rsc-advances

\section{Enhanced thermal properties of poly(vinylidene fluoride) composites with ultrathin nanosheets of MXene†}

\author{
Yong Cao,,$^{\mathrm{a}}$ Qihuang Deng, $\ddagger^{\mathrm{b}}$ Zhiduo Liu, ${ }^{\mathrm{a}}$ Dianyu Shen, ${ }^{a}$ Ting Wang, ${ }^{\mathrm{a}}$ Qing Huang, ${ }^{\mathrm{b}}$

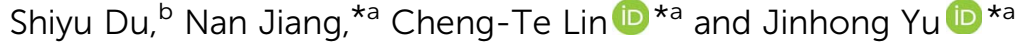

\begin{abstract}
Here, we report a facile method to delaminate $M X e n e\left(\mathrm{Ti}_{3} \mathrm{C}_{2} \mathrm{~T}_{x}\right)$ and prepare poly(vinylidene fluoride) (PVDF)/MXene composites by solution blending. Compared with neat PVDF, the PVDF composites with varying content of MXene (0-5 wt\%) showed an enhancement in the PVDF thermal conductivity. In particular, when a loading of $5 \mathrm{wt} \%$ was attained, the thermal conductivity was increased to $0.363 \mathrm{~W}$ $\mathrm{mK}^{-1}$, an approximate 1-fold enhancement compared with that of neat PVDF. In addition, MXene also exhibited a better performance in enhancing the thermal dynamic mechanical properties of PVDF. For instance, PVDF composites with only 5 wt\% MXene exhibited a storage modulus as high as $7501 \mathrm{MPa}$, corresponding to a $64 \%$ enhancement compared with that of neat PVDF. In light of the excellent thermal properties of the PVDF/MXene composites, they can be expected to have a wide range of potential applications in thermal interfacial materials and structural components.
\end{abstract}

\section{Introduction}

Since the first discovery of two-dimensional (2D) layers composed of transition metal carbides and carbonitrides in 2011, these materials have attracted increasing attention from scientists due to their combined metallic conductivity and hydrophilic surfaces. ${ }^{1}$ They are often labelled "MXenes", produced by etching the " $\mathrm{A}$ " element from " $\mathrm{M}_{n+1} \mathrm{AX}_{n}$ phases", where " $M$ " stands for a transition metal, "A" is a group (mainly referring to group IIIA or IVA element), " $\mathrm{X}$ " represents carbon or nitrogen atoms and $n=1,2,3 .^{2} \mathrm{Up}$ to now, several MXenes have been successfully synthesized, such as $\mathrm{Ti}_{3} \mathrm{C}_{2}, \mathrm{Ti}_{2} \mathrm{C}$, $\left(\mathrm{Ti}_{0.5}, \mathrm{Nb}_{0.5}\right)_{2} \mathrm{C},\left(\mathrm{V}_{0.5}, \mathrm{Cr}_{0.5}\right)_{3} \mathrm{C}_{2}, \mathrm{Ti}_{3} \mathrm{CN}, \mathrm{Ta}_{4} \mathrm{C}_{3},{ }^{3} \mathrm{Nb}_{2} \mathrm{C}, \mathrm{V}_{2} \mathrm{C},{ }^{4} \mathrm{ZrC},{ }^{5}$ and $\mathrm{Nb}_{4} \mathrm{C}_{3} .{ }^{6}$ Among these MXenes, $\mathrm{Ti}_{3} \mathrm{C}_{2}$, which is readily obtained by selectively etching and exfoliating the Al layers from $\mathrm{Ti}_{3} \mathrm{AlC}_{2}$ ceramics with $\mathrm{HF}$, has drawn widespread public attention and research. ${ }^{7}$ Given that MXene surfaces are terminated by $\mathrm{O}, \mathrm{OH}$, and/or $\mathrm{F}$ groups due to $\mathrm{HF}$ etching, MXenes $\left(\mathrm{Ti}_{3} \mathrm{C}_{2}\right)$ can be represented as $\mathrm{Ti}_{3} \mathrm{C}_{2} \mathrm{~T}_{x}$, where $\mathrm{T}$ stands for the terminal

${ }^{a}$ Key Laboratory of Marine Materials and Related Technologies, Zhejiang Key Laboratory of Marine Materials and Protective Technologies, Ningbo Institute of Materials Technology \& Engineering, Chinese Academy of Sciences, Ningbo, 315201, China.E-mail: Jiangnan@nimte.ac.cn; linzhengde@nimte.ac.cn; yujinhong@nimte. ac.cn

${ }^{b}$ Division of Functional Materials and Nanodevices, Ningbo Institute of Materials Technology and Engineering, Chinese Academy of Sciences, Ningbo, Zhejiang, 315201, China

† Electronic supplementary information (ESI) available. See DOI: 10.1039/c7ra00184c

\$ These authors contributed equally to this study. group $(\mathrm{O}, \mathrm{OH}$, and/or $\mathrm{F})$ and " $x$ " refers to the number of terminal groups.

Generally speaking, MXenes are not delaminated and exhibit multilayered structures similar to exfoliated graphite. Based on previous reports, MXenes also show great promise in many applications, owing to their excellent inherent physiochemical properties. These applications include electrodes for electrochemical supercapacitors, ${ }^{8-10}$ promoters for catalysts, ${ }^{\mathbf{1 1}, 12}$ and absorbents for heavy metal ions. ${ }^{13}$ To further exploit the unique properties of MXenes $\left(\mathrm{Ti}_{3} \mathrm{C}_{2} \mathrm{~T}_{x}\right)$, much effort has been dedicated to delaminating multilayered MXenes. ${ }^{7,10,14}$ It is well known that graphene has prominent thermal conductivity and mechanical strength, namely, $3000 \mathrm{~W} \mathrm{mK^{-1 }}$ and $1060 \mathrm{GPa}$, respectively, ${ }^{15,16}$ while those of ultrathin boron nitride (BN) are theoretically estimated to be $2000 \mathrm{~W} \mathrm{mK}{ }^{-1}$ and $800 \mathrm{GPa},{ }^{17}$ respectively. Due to their impressive properties, graphene and $\mathrm{BN}$ are often utilized as a promising fillers in polymer composites to enhance their thermal and mechanical properties. ${ }^{\mathbf{1 8 - 2 2}}$ Similar to graphene and $\mathrm{BN}$, two dimensional MXenes have good physical properties, such as mechanical strength, electrical conductivity and thermal conductivity. ${ }^{23-28}$ Some previous studies have reported the incorporation of MXenes $\left(\mathrm{Ti}_{3} \mathrm{C}_{2} \mathrm{~T}_{x}\right)$ into different polymer matrices, such as ultrahigh-molecular polyethylene (UMWPE), polypyrrole (PPy), and polyvinyl alcohol (PVA). ${ }^{29-32}$ Therefore, the inclusion of inorganic MXenes into polymer matrices for the formation of composites has proved to be an efficient way to obtain multifunctional polymer composites.

In this study, $\mathrm{Ti}_{3} \mathrm{AlC}_{2}$ was chosen as the MAX precursor because its exfoliation and delamination have already been developed previously. ${ }^{33-35}$ Poly(vinylidene) fluoride (PVDF) is 
a semi-crystalline polymer with five different crystalline forms (namely $\alpha, \beta, \gamma, \delta$ and $\varepsilon$ ). ${ }^{36,37}$ Due to its excellent piezoelectric, pyroelectric and ferroelectric properties, PVDF has applications in many fields, including sensors, actuators, membranes and smart scaffolds. ${ }^{38-42}$ However, the thermal conductivity of PVDF is low, which limits its use in fields where heat transfer is important. Therefore, we chose PVDF as a polymer matrix and used ultrathin MXene as a filler to fabricate PVDF/MXene composites by simple solution blending and hot pressing. The as-fabricated PVDF/MXene composites showed better thermal properties, which have not been reported before, to the best of our knowledge.

\section{Experimental}

\subsection{Materials}

PVDF powder was purchased from $3 \mathrm{~F}$ Co. Ltd, Shanghai (China). $\mathrm{Ti}_{3} \mathrm{AlC}_{2}$ power was purchased from Forsman Scientific Co. Ltd., Beijing (China). N,N-Dimethylformamide (DMF) with a purity of $99.5 \%$ was purchased from Sinopharm Chemical Reagent Co. Ltd, Shanghai (China), and was used without further purification. HF was also purchased from Sinopharm Chemical Reagent Co. Ltd., Shanghai (China).

\subsection{Preparation of PVDF/MXene composites}

$\mathrm{Ti}_{3} \mathrm{AlC}_{2}$ power $(10 \mathrm{~g})$ was immersed in $100 \mathrm{ml}$ of a $40 \% \mathrm{HF}$ solution. The mixture was stirred for 3 minutes, and then kept at room temperature for $2 \mathrm{~h}$. The as-prepared suspension was then washed using deionized water several times and centrifuged to separate the powder when the $\mathrm{pH}$ of the suspension was higher than 6 . Then the washed powders were filtered under vacuum conditions and dried at room temperature for $24 \mathrm{~h}$. Properly dried MXene $\left(\mathrm{Ti}_{3} \mathrm{C}_{2}\right)$ determined by experiment was added into $100 \mathrm{ml}$ DMF solution and was then ultrasonicated for $1 \mathrm{~h}$ to form a homogeneously dispersed suspension, which mainly consisted of ultrathin MXene nanosheets. Meanwhile, the corresponding PVDF powder was entirely dissolved in $30 \mathrm{ml}$ DMF and poured into the MXene suspension. The mixture was stirred with an electric stirrer at a speed of $3000 \mathrm{rpm} \mathrm{min}^{-1}$ while the distillation process was performed simultaneously at a temperature of $180^{\circ} \mathrm{C}$. It should be noted that when the distillation process had finished, the residue was put into a vacuum oven and dried at $100{ }^{\circ} \mathrm{C}$ for $5 \mathrm{~h}$ to remove the residual DMF. Finally, the dried mixture was compressed to form samples of the required size, by hot pressing under a pressure of about $10 \mathrm{MPa}$. The detailed preparation process is vividly exhibited in Scheme 1.

\subsection{Characterization}

The X-ray diffraction (XRD) patterns of the specimens were collected using a D8 DISCOVER with GADDS (BRUKER Ltd. Germany), at a scan rate of $10{ }^{\circ} \mathrm{C} \mathrm{min}{ }^{-1}$ and $2 \theta$ ranging from $5^{\circ}$ to $60^{\circ}$, using $\mathrm{Cu} \mathrm{K} \alpha$ radiation $(\lambda=1.5406 \AA)$ at room temperature. The Raman spectra were recorded using a Reflex Raman system (RENISHAW plc, Wotton-under-Edge, UK) with a laser wavelength of $532 \mathrm{~nm}$. As-prepared MXenes and delaminated

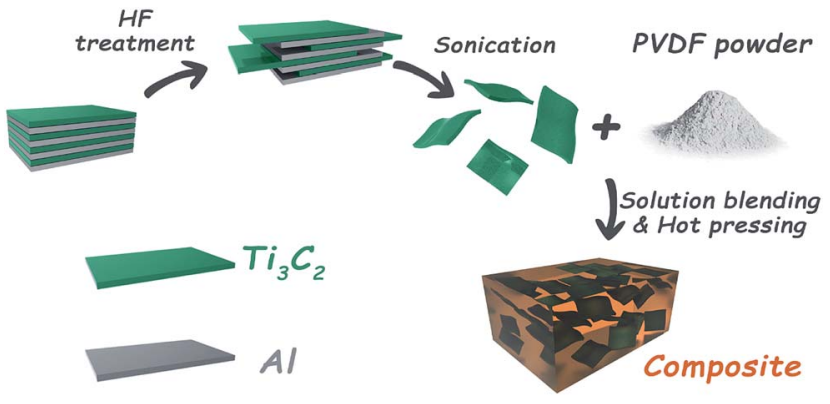

Scheme 1 The preparation process of PVDF/MXene composites.

MXene nanosheets in DMF were characterized using a UV/VIS/ NIR spectrophotometer (PerkinElmer, Lambda 950, U.S.A.). Atomic force micrographs (AFM) were obtained using a Nanoscope III scanning probe microscope (Dimension 3100, Veeco, USA) in tapping mode. X-ray photoelectron spectroscopy (XPS using an AXIS Ultra DLD spectrometer, Kratos, Japan) was used to analyze the surfaces of the samples. A field emission scanning electron microscope (FE-SEM, QUANTA FEG250, U.S.A.) was used to obtain images of the MXenes and the fractured surfaces of PVDF samples with an accelerating voltage of $20 \mathrm{kV}$. The fractured surfaces were first sputtered with a thin layer of gold to avoid the accumulation of charge. The microstructures of ultrathin MXene nanosheets were observed using a JEOL JEM-2100 (Electron Optics Laboratory Co., Ltd, Japan) instrument with an acceleration voltage of $200 \mathrm{kV}$, and samples for TEM measurements were fabricated by drop casting onto carbon-coated copper grids followed by solvent evaporation in air. The thermal conductivities of the composites were measured using an LFA 447 Nanoflash (NETZSCH, Germany). Differential scanning calorimetry (DSC) experiments were carried out under nitrogen on a Pyris Diamond DSC (PerkinElmer, U.S.A.). Thermogravimetric analysis (TGA) was performed on a TGA 209 F3 (NETZSCH, Germany). The samples were close to $10 \mathrm{mg}$ and all the measurements were performed under a nitrogen atmosphere. Dynamic mechanical analysis (DMA) was performed on a DMA Q800 dynamic mechanical analyzer (TA instruments, U.S.A.), operating in the tension mode at an oscillation frequency of $1 \mathrm{~Hz}$. The IR-photos were captured by an infrared camera (Fluke, Ti400, U.S.A.).

\section{Results and discussion}

\subsection{Characterization of MXene}

The FE-SEM images of un-delaminated and delaminated MXene are shown in Fig. 1(a) and (b), respectively. From Fig. 1(a), one can observe that un-delaminated MXene possesses multilayers. The inset further shows that the thickness of the layered structure is small and there are some spacings of different sizes between the layers. Fig. 1(b) exhibits the morphology of delaminated MXene on a silicon substrate. Obviously, the multilayered MXene possesses a flake-like structure, indicating that the exfoliation process had been essentially completed. The inset is a high-resolution image of 

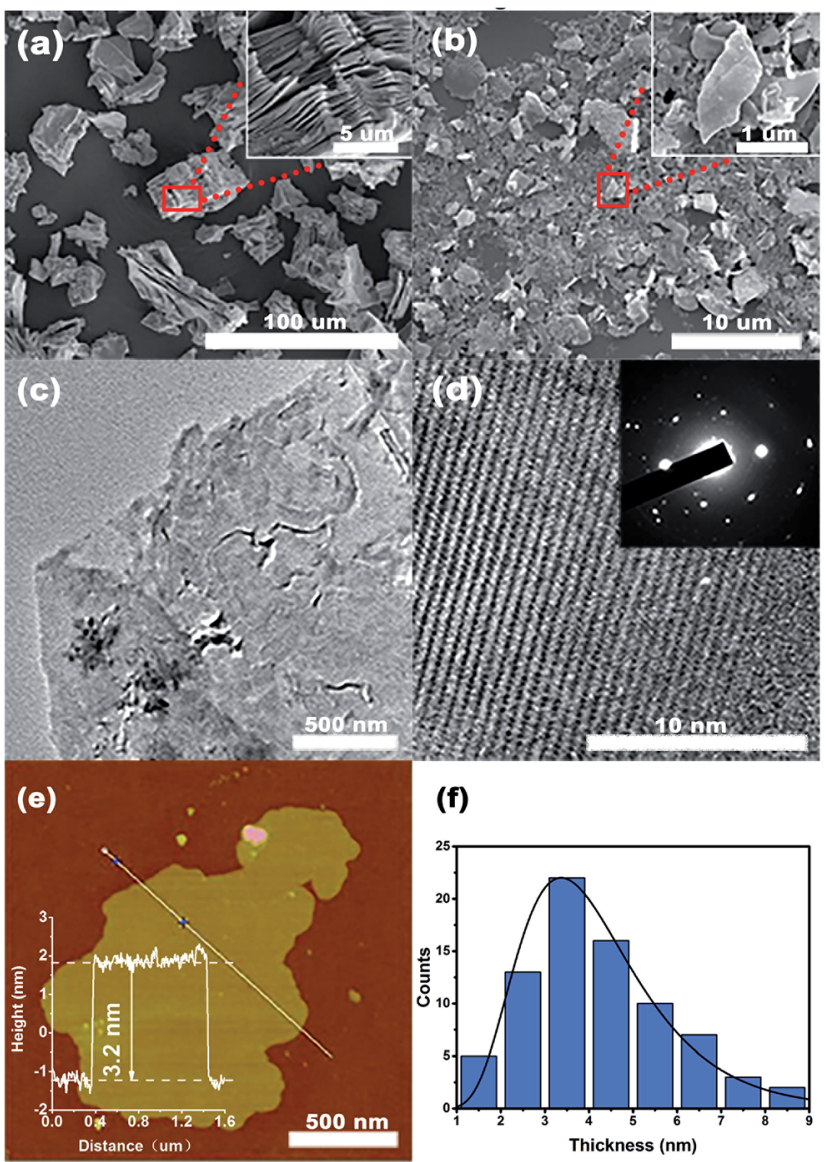

(f)

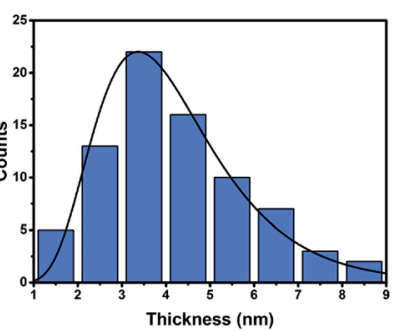

Fig. 1 FE-SEM images of (a) un-delaminated MXene and (b) delaminated MXene. The insets in (a) and (b) are high-resolution FE-SEM images exhibiting the morphologies of un-delaminated and delaminated MXene. (c) TEM and (d) high-resolution electron microscopy images of delaminated MXene. The inset in (d) shows the selected area electron diffraction (SAED) pattern. (e) The AFM image of exfoliated MXene and the corresponding height measurement. (f) Counts at different thicknesses of MXene nanosheets.

the selected area marked with a red rectangle in Fig. 1(b). Based on the inset, it is easy to figure out that the lateral size of the exfoliated MXene is close to $1 \mu \mathrm{m}$. TEM images of the delaminated MXene sheets proved them to be very thin and transparent, as shown in Fig. 1(c). Furthermore, there are some wrinkles on the sheets, which may be due to the flexibility of the MXene nanosheets. Fig. 1(d) is a high-resolution TEM (HRTEM) image of a typical MXene nanosheet and its corresponding selected area electron diffraction (SAED) pattern (inset in Fig. 1(c)). The SAED pattern shows the hexagonal symmetry of the nanosheet, which reveals that the as-delaminated nanosheets are single crystals. Fig. 1(e) shows an AFM image of a single MXene nanosheet and its corresponding height measurement. One can clearly observe that the MXene nanosheet is very flat and very thin, with a thickness of $3.2 \mathrm{~nm}$. In order to figure out the average thickness of the MXene nanosheets, we performed a statistical analysis of 78 pieces, and the plot is shown in Fig. 1(f). From this diagram, we can see that the thicknesses of the MXene nanosheets range from 1 to $9 \mathrm{~nm}$, with a mean thickness of $4.1 \mathrm{~nm}$. For nanosheets with thicknesses of 2-6 nm, the probability of their occurrence is obviously increased, indicating that the delamination of MXenes is efficient.

Fig. 2(a) shows the Raman spectrum of the MXene. One can clearly observe that there are four sharp peaks occurring at 215, 375, 612 and $698 \mathrm{~cm}^{-1}$, which are indicative of MXene. ${ }^{7}$ Furthermore, there are also some inconspicuous peaks, such as those at 167,246 and $570 \mathrm{~cm}^{-1}$. Similar to $\mathrm{Ti}_{3} \mathrm{SiC}_{2}$, the peaks at 167,215 and $235 \mathrm{~cm}^{-1}$ can be assigned to Al-Ti vibrations, while the peaks at around 375,612 and $698 \mathrm{~cm}^{-1}$ conform with Ti-C bond vibrations..$^{43}$ It is worth noting that there is a small sharp peak at around $150 \mathrm{~cm}^{-1}$. The reason for this may be because the laser power was too high, leading to the formation of oxidized $\mathrm{Ti}_{3} \mathrm{C}_{2}$.

A typical XRD pattern of the MXene is shown in Fig. 2(b). Peaks at $2 \theta$ values of $8.3,18.3,23.2,27.5,34.1,36.2,39.0,41.7$, 50.8 and $52.7^{\circ}$ are assigned to the (002), (004), (006), (008), (101), (103), (104), (105), (107) and (108) planes, respectively. Among these, (002) (004) (006) (008) and (105) are indicative of MXene, while other crystal planes are present due to $\mathrm{Ti}_{3} \mathrm{AlC}_{2}$. It has been reported that the characteristic (002) peak of $\mathrm{Ti}_{3} \mathrm{AlC}_{2}$ is at $9.5^{\circ}$, but in this work it is shifted to a lower value $\left(8.3^{\circ}\right)$. This is considered to be due to structural expansion from etching and the substitution of $\mathrm{Al}$ with $-\mathrm{F}$ and $-\mathrm{OH} /=\mathrm{O}$ terminating groups, resulting in a large $d$-spacing. Furthermore, the intense peak at $2 \theta=39^{\circ}$ is much lower, and is not easily perceived in comparison to other examples in the literature, ${ }^{44}$ indicating that Al layers in $\mathrm{Ti}_{3} \mathrm{AlC}_{2}$ are removed in a more effective way. ${ }^{45,46}$ Fig. 2(c) shows the thermal stability of MXene from 50 to $600{ }^{\circ} \mathrm{C}$ in nitrogen. It shows that the MXene, as a whole, has a relatively good thermal stability. Furthermore, it should be noted that a big weight loss occurs at $125^{\circ} \mathrm{C}$, attributed to MXene powder absorbing water in the air, which then evaporates beyond $100{ }^{\circ} \mathrm{C}$. Meanwhile, high-temperature heat treatment may also
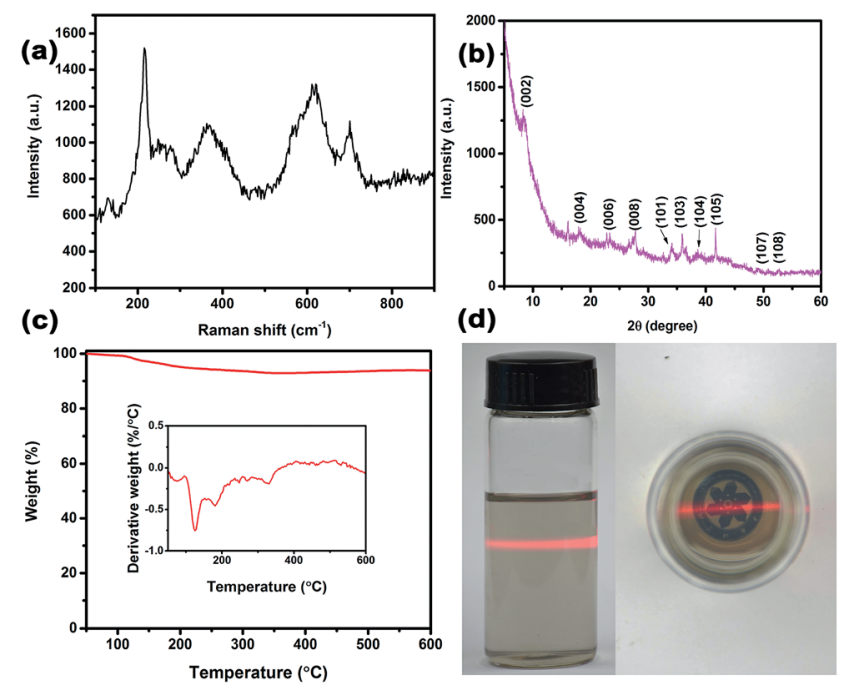

Fig. 2 (a) Raman spectra and (b) XRD pattern of un-delaminated MXene. (c) TGA and DTG curve of MXene. (d) Tyndall effect of MXene nanosheets dispersed in DMF. 
result in the absence of groups containing $-\mathrm{F}$ or $-\mathrm{OH} /=\mathrm{O}$. Fig. 2(d) depicts an interesting experimental phenomenon, in which an obvious passage appeared when a light beam from a laser pointer was passed through a homogeneously dispersed solution of MXene in $\mathrm{N}, \mathrm{N}$-dimethylformamide (DMF). This confirms the Tyndall effect, whereby a light beam traversing a colloidal solution leaves an identifiable scattering track in the suspension. It also implies the existence of ultrathin MXene nanosheets in DMF. In addition, the MXene nanosheets in DMF and the UV/visible absorption spectra are shown in Fig. S1.†

To obtain more information about the surface chemistry of the MXene, we carried out XPS analysis and the results are shown in Fig. 3. The survey showed obvious signals from Ti, C, $\mathrm{O}$, and F elements (Fig. 3(a)). Specifically, peaks at binding energy values of $33,120,284,453,476,530,557,593,682,734$, 832 and $985 \mathrm{eV}$ are assigned to O2s, Al2s, C1s, Ti2p, Ti2p1/2, O1s, Ti2s, F KLL, F1s, O KLL, Ti LMM and C KLL, respectively, which is in good agreement with the EDAX results shown in Fig. S2. $\dagger$ It also revealed the existence of a small amount of Al, which was most likely derived from the residue after the $\mathrm{Ti}_{3} \mathrm{AlC}_{2}$ etching reaction. High-resolution XPS spectra in the C1s region (Fig. 3(b)) of the MXene powder revealed three main peaks occurring at $281.38,284.60$ and $287.76 \mathrm{eV}$, which may originate from $\mathrm{Ti}-\mathrm{C}, \mathrm{C}-\mathrm{C}$ and $\mathrm{C}-\mathrm{O}$, respectively. The presence of $\mathrm{C}-\mathrm{O}$ may arise from MXene oxidation, resulting in the formation of $\mathrm{TiO}_{2}$ and carbon atom networks. ${ }^{47}$ Fig. $3(\mathrm{c})$ shows the high-resolution
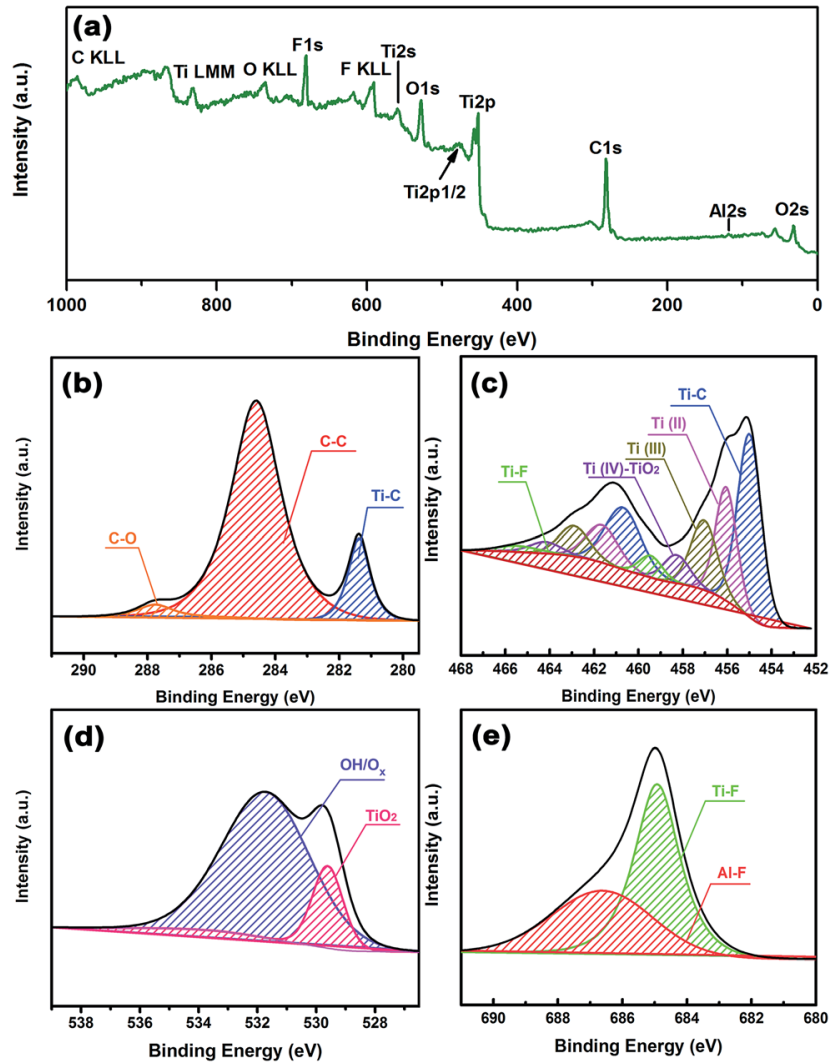

Fig. 3 XPS spectra of MXene powder: (a) survey, (b-e) high resolution spectra of $\mathrm{C} 1 \mathrm{~s}, \mathrm{Ti2p}, \mathrm{O} 1 \mathrm{~s}$, and F1s regions, respectively.
XPS spectra of the Ti2p region, which possess peaks that are deconvoluted into components corresponding to Ti bound to C, and $\mathrm{Ti}$ (II), $\mathrm{Ti}(\mathrm{III}), \mathrm{Ti}(\mathrm{IV})$ and $\mathrm{Ti}$ bound to $\mathrm{F}$. It is believed that the $\mathrm{Ti}-\mathrm{C}$ signal results from $\mathrm{Ti}$ atoms in the interior of the MXene layers. ${ }^{\mathbf{1 4 , 4 8 , 4 9}}$ With regard to the Ti signals with oxidation states, such as Ti(II), Ti(III) and Ti(Iv), these may arise from the formation of mixed oxides $\left(\mathrm{TiO}_{x} \mathrm{~F}_{y}\right)$ and carboxides $\left(\mathrm{TiC}_{x} \mathrm{O}_{y}\right)$, indicating that the termination of the MXene surface is not uniform. The high-resolution spectrum in the 01s regions (shown in Fig. 3(d)) confirms the formation of Ti(rv) oxide, and demonstrates the presence of $\mathrm{OH} / \mathrm{O}_{x}$ groups, which are likely bound to the MXene surfaces. From Fig. 3(e), one can clearly observe Ti-F and Al-F signals, confirming the presence of $\mathrm{F}$ groups caused by HF etching. In summary, XPS analysis comprehensively clarified the surface composition and functional groups, such as $-\mathrm{F},-\mathrm{OH}$ and $-\mathrm{O}_{x}$, highlighting the variation and complexity in the chemistry of the MXene material.

\subsection{Morphology of composites}

It is known to us that the dispersion of filler in the matrix and the interaction between the matrix and filler are crucial for improving the various properties of the PVDF matrix. In order to investigate the effect of MXene on the comprehensive properties of the PVDF composites, the morphology of the fractured

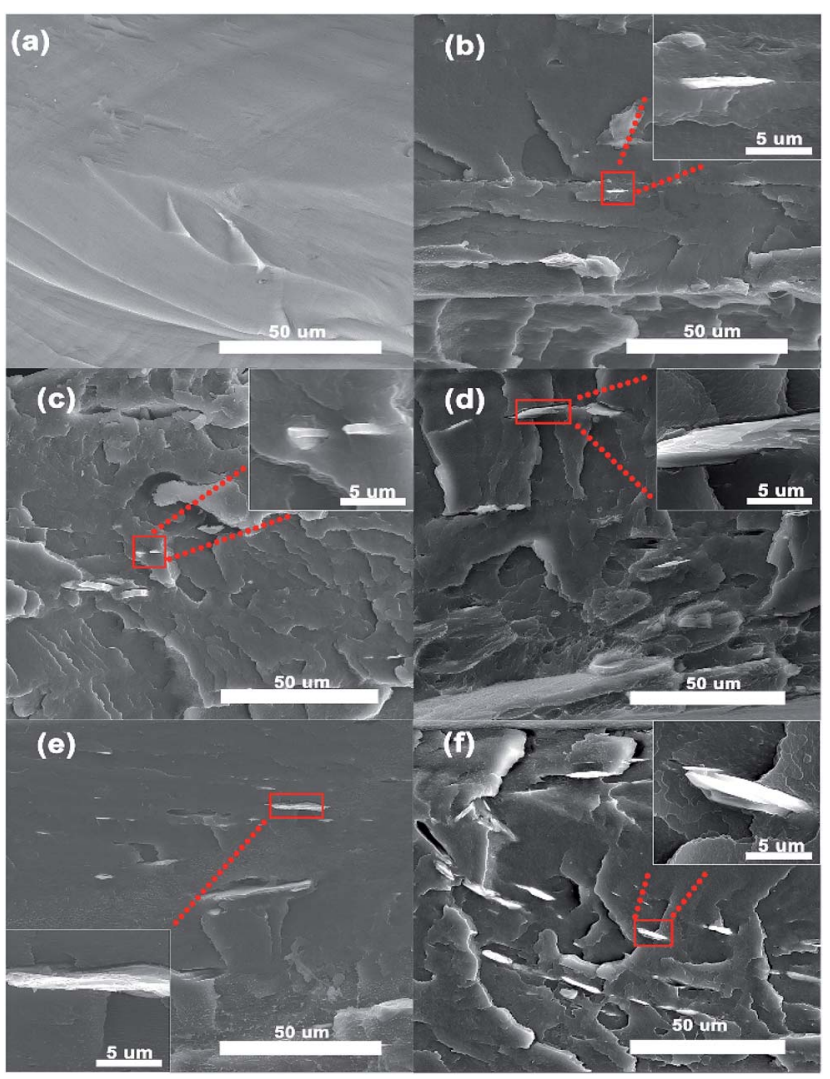

Fig. 4 FE-SEM images of the fractured surface of (a) neat PVDF and $(b-f)$ PVDF composites containing different MXene loadings. The insets in $(b-f)$ are the high-resolution FE-SEM images showing the morphologies of MXene in the PVDF matrix. 
surfaces (including neat PVDF) was characterized using FESEM, as shown in Fig. 4. Fig. 4(a) shows that the fractured surface of neat PVDF is very smooth with only a few cracks, which is representative of polyolefin. It is believed that the presence of cracks is because the surface failed to elastically release in the context of external force. Fig. 4(b)-(f) show the FESEM photographs of the PVDF composites containing 1, 2, 3, 4 and $5 \mathrm{wt} \%$ of MXene, respectively, and their corresponding high-resolution FE-SEM images. Obviously, the fractured surfaces of the PVDF composites are rougher compared with those of neat PVDF. Furthermore, MXene is homogenously dispersed and embedded in the matrix, as shown in Fig. 4(b)(f), indicating good compatibility between the filler and matrix. With an increase in the loading of MXene, one can clearly see more and more thin and thick MXene nanosheets with different shapes emerge at the surface. For instance, the insets in Fig. 4(d) and (e) explicitly show the morphology of ultrathin MXene sheets in the matrix, which is consistent with Fig. 1(b). Additionally, Fig. S3† indicates that the inclusion of MXene nanosheets into the PVDF matrix does not lead to a crystal transition, further proved by the DSC curves (shown in Fig. S4†). This is different from other two-dimensional materials. ${ }^{\mathbf{5 0}}$

\subsection{Thermal properties of composites}

Fig. 5(a) shows the thermal diffusivity and thermal conductivity of neat PVDF and the PVDF composites with different filler content. It is obvious that both the thermal conductivity and the thermal diffusivity monotonically increase with the addition of MXene. To be specific, the thermal diffusivity values of neat PVDF and the PVDF composites with 1, 2, 3, 4 and 5 wt $\%$
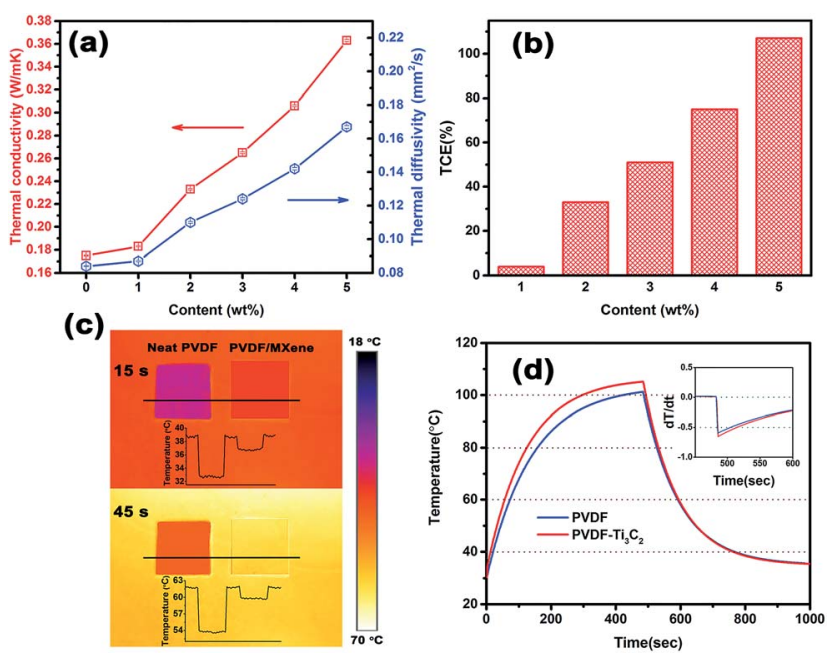

Fig. 5 (a) Thermal diffusivity and thermal conductivity of neat PVDF and PVDF composites with different filler loadings. (b) Thermal conductivity enhancement (TCE) of PVDF composites compared to neat PVDF. (c) Infrared images of neat PVDF, and PVDF composites with 5 wt\% MXene. The temperature gradient scale bar on the right shows the highest and lowest temperatures of $70{ }^{\circ} \mathrm{C}$ and $18{ }^{\circ} \mathrm{C}$, respectively. (d) Corresponding surface temperature variation with time upon heating and cooling. Cooling started at 482 seconds for each sample. loading are $0.084,0.087,0.11,0.124,0.142$ and $0.167 \mathrm{~mm}^{2} \mathrm{~s}^{-1}$, respectively, corresponding to thermal conductivity values of $0.175,0.183,0.233,0.265,0.306$ and $0.363 \mathrm{~W} \mathrm{mK}^{-1}$. Compared with neat PVDF, the PVDF composites exhibit better heat dissipation. As can be seen in Fig. 5(b), the thermal conductivity of the PVDF composite containing $5 \mathrm{wt} \%$ MXene achieved the maximum thermal enhancement of approximately 1-fold, in comparison to neat PVDF, while the other corresponding compositions reached 4, 33, 51 and 75\% enhancements, respectively. In order to investigate the thermal conductivity of the PVDF/MXene composites as compared with other thermal interface materials, we summarized the reported thermal conductivity values of thermal interface materials with various thermal conductive fillers, as shown in Table 1 . The data demonstrates that the thermal conductivity (TC) in our PVDF/ MXene composites is comparable to or higher than that of various thermal conductive fillers in polymer matrices reported in previous works. ${ }^{42-52}$ It is worth noting that in terms of enhancing the thermal conductivity of the polymer, the composite with $5 \mathrm{wt} \%$ MXene surpasses GNP with the same loading. It is known to us that the thermal conductivity of a polymer composite is significantly affected by the loading, structure, dispersion of fillers, and thermal interfacial resistance between filler and matrix. As for the PVDF/MXene composites, when the loading is $1 \mathrm{wt} \%$, the thermal conductivity increases from 0.175 to $0.183 \mathrm{~W} \mathrm{mK}^{-1}$, indicating that the improvement is not obvious. As the content gradually increases, the thermal conductivity achieves a large improvement. The reason behind this improvement in the thermal conductivity may be explained as follows: when the loading of MXene is low, for instance, $1 \mathrm{wt} \%$, MXene can serve as a bridge between PVDF spherulites and promote heat transfer from spherulites to spherulites. Since the sea-island structure of PVDF/MXenes composites does not attain a percolation threshold of heat transportation, it is difficult for MXene in the PVDF matrix to form a continuous network, resulting in only a slight increase in the thermal conductivity. When the addition of MXene is increased and MXene nanosheets are well dispersed in the PVDF matrix, the thermal conductivity of PVDF composites will increase at a rapid rate. The situation may be attributed to several factors. Firstly, MXene nanosheets are uniformly dispersed in the PVDF matrix and the contact ratio between them is increased. Secondly, MXene in the matrix can provide nucleation sites for the PVDF matrix, which is crucial for improving the thermal conductivity of composites. Finally, the strong interface compatibility between MXene and PVDF matrix reduces the interfacial thermal resistance, due to the large surfaces of the MXene nanosheets and hydrogen bonding between MXene and the PVDF matrix.

In order to visually verify the heat performance of neat PVDF and the PVDF composite containing 5 wt $\%$ MXene, we employed an infrared camera to record the different thermal states of the samples at different times, as shown in Fig. 5(c). Several steps needed to be taken before the experiment. First, clean and flat copper foil was placed on the heating plate, and then a high temperature adhesive tape was used to fix the copper foil and ensure that it was flat on the surface of the 
Table 1 Thermal interface materials with various thermal conductive fillers

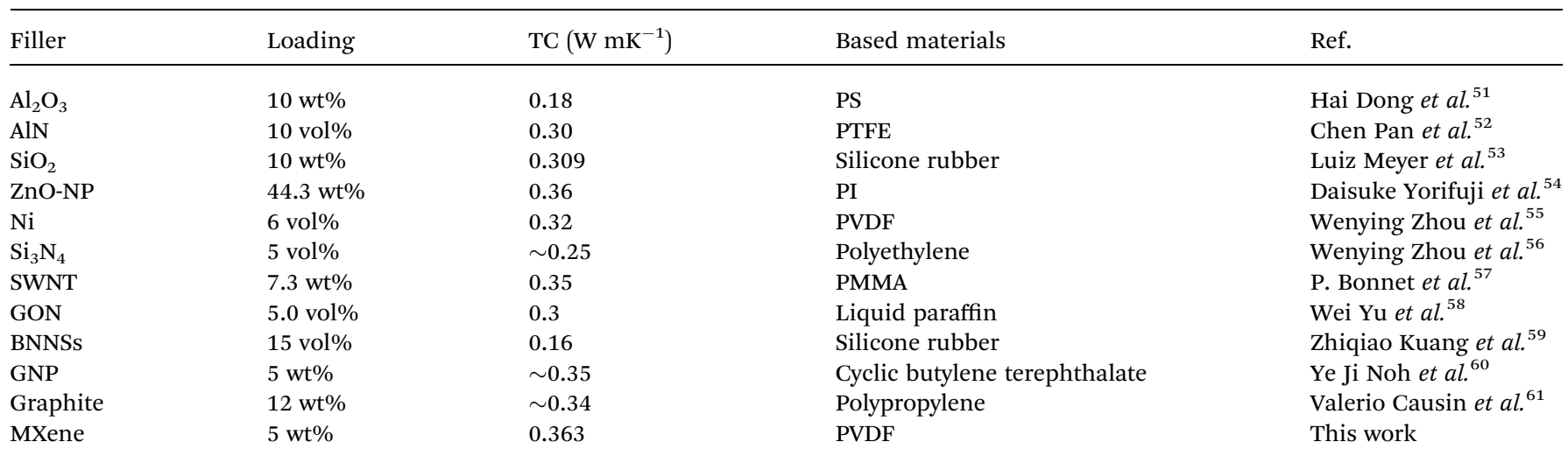

heating plate. Finally, the two samples were placed in order, as shown in Fig. 5(c). It is should be noted that the samples and copper foil were sprayed with graphite in order to maintain heat transfer in a uniform way. When everything necessary was done, we started to heat the heater and record the thermal states of the samples using an infrared camera. It can be clearly seen that the surface color of the samples changed as the temperature of the heater increased instantly. This is especially true for the PVDF composite, as its color looks much brighter than that of neat PVDF. Furthermore, we performed a temperature statistical analysis of 70 dots from the black line and plotted a diagram to show the temperature of the samples. From the upper diagram, one can find the surface temperature of neat PVDF was around $33{ }^{\circ} \mathrm{C}$, while those of PVDF/MXene and the copper foil were around 37 and $39^{\circ} \mathrm{C}$, respectively. At $45 \mathrm{~s}$, one can observe that the surface temperature of neat PVDF was about $54{ }^{\circ} \mathrm{C}$ and the average temperature of the PVDF/MXene composite was $60{ }^{\circ} \mathrm{C}$, indicating that the heating rate of the PVDF composite is faster than that of neat PVDF. This conclusion is also in agreement with Fig. 5(a). Fig. 5(d) shows the quantitative heating and cooling curves measured using a versatile voltmeter. It is evident that the heating curve of the PVDF composite is always above that of neat PVDF and reaches its highest temperature at $104{ }^{\circ} \mathrm{C}$, while neat PVDF reaches a maximum at $101{ }^{\circ} \mathrm{C}$, demonstrating that PVDF composites possess a higher heating rate. To further investigate the release performance of neat PVDF and the PVDF composite, we processed the cooling curve with differential treatment and the result is shown in the inset of Fig. 5(d). From the inset, it is easy to see that the cooling rate of the PVDF composite is faster than that of neat PVDF, and is the first to reach room temperature. To sum up, MXene has the potential to enhance the thermal conductivity of the PVDF matrix and can be regarded as a promising candidate for thermal management.

Fig. 6 displays the thermal dynamic mechanical properties of the neat PVDF and PVDF composites as a function of temperature measured by DMA Q800. As can be seen from Fig. 6(a), the addition of MXene nanosheets into the neat PVDF matrix results in an increase in the storage modulus of the glassy state. The storage modulus of neat PVDF at $-80{ }^{\circ} \mathrm{C}$ is $4.362 \mathrm{GPa}$, while the values for PVDF/MXene with loadings from 1 to $5 \mathrm{wt} \%$ are
5.491, 5.591, 5.831, 6.136 and $7.128 \mathrm{GPa}$, respectively, corresponding to increases of $26 \%, 28 \%, 34 \%, 40 \%$ and $64 \%$, respectively, compared to neat PVDF. The storage modulus represents the elastic modulus of a plastic material and its ability to recover. It is observed that the PVDF/MXene composites have a higher storage modulus than that of neat PVDF, which increases when the loading increases from 1 to 5 $\mathrm{wt} \%$. There are two reasons that may account for the improvement in the storage modulus properties. First, due to the high surface area and good dispersion in the PVDF matrix, MXene nanosheets enhance the stiffness of the composites and make it possible for the applied stresses to transfer from the PVDF matrix to the MXene nanosheets. Second, MXene forms a strong interaction with the PVDF matrix as a result of the functional groups, which hinders the mobility of the local matrix around MXene. Fig. 6(b) shows the storage modulus ratio of the PVDF/ MXene composites ( $\left.E_{\text {composites }}^{\prime \prime}\right)$ to neat PVDF ( $\left.E_{\text {neat PVDF}}^{\prime}\right)$. It is evident that the relative storage modulus ratios of the PVDF/ MXene composites are above 1 and gradually increase with increasing loading. When the loading is $5 \mathrm{wt} \%$, the $E^{\prime \prime}$ composites $/$
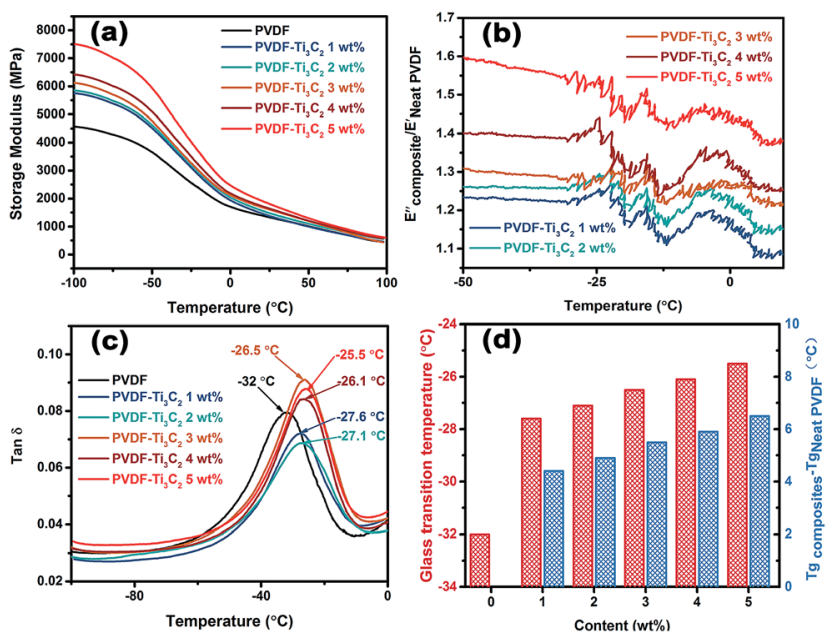

Fig. 6 Thermo-mechanical properties of neat PVDF and the PVDF composites: (a) storage modulus, (b) relative storage modulus, (c) tan $\delta$ as a function of temperature, and (d) glass transition temperature. 
$E^{\prime}$ neat PVDF attains a maximum value of 1.6 at $-50{ }^{\circ} \mathrm{C}$. In addition, the melting behavior and thermal stability of the PVDF/ MXene composites are shown in Fig. S4 and S5. $\dagger$ Fig. 6(c) shows the loss factors of neat PVDF and the PVDF composites as a function of temperature. The loss factor $\tan \delta$ refers to the ratio of the loss modulus to the storage modulus, which is quite sensitive to transformations in material structure. Furthermore, the peak value of the $\tan \delta$ curve determines the glass transition temperature $\left(T_{\mathrm{g}}\right)$ of the composites. As can been seen from Fig. 6(c), the $T_{\mathrm{g}}$ of the neat PVDF is $-32{ }^{\circ} \mathrm{C}$, while the values for the PVDF/MXene composites containing different MXene contents are $-27.6,-27.1,-26.5,-26.1$, and $-25.5{ }^{\circ} \mathrm{C}$, respectively. Obviously, the PVDF/MXene composites show an increased $T_{\mathrm{g}}$ compared to neat PVDF. The increase in $T_{\mathrm{g}}$ is believed to result from the restriction in molecular motion and the higher degree of crosslinking, leading to obvious changes in polymer chain dynamics. ${ }^{\mathbf{6 2}, \mathbf{6 3}}$ For the PVDF composites, the incorporation of MXene hinders the molecular mobility. On the other hand, the presence of functional groups on the surface of MXene nanosheets improves the interfacial interaction between MXene and the PVDF matrix, resulting a good uniform dispersion within the matrix, which is beneficial for achieving a big shift in $T_{\mathrm{g}}$. Fig. 6(d) shows the $T_{\mathrm{g}}$ values of neat PVDF and PVDF/ MXene and presents the increasing values compared to neat PVDF. The red bar represents the $T_{\mathrm{g}}$ of neat PVDF and the PVDF composites, while the blue bar shows the enhancement in the $T_{\mathrm{g}}$ value, that is, $4.4{ }^{\circ} \mathrm{C}$ for the PVDF composite with $1 \mathrm{wt} \%$ MXene, and 4.9, 5.5, 5.9 and $6.5{ }^{\circ} \mathrm{C}$ for the PVDF composites with 2, 3, 4 and $5 \mathrm{wt} \%$, respectively. Given that the PVDF/MXene composites exhibit better thermo-mechanical properties, they can be expected to perform well in a wide range of applications, such as sensors and actuators, separators, batteries, filtration membranes and smart scaffolds. ${ }^{38-42}$

\section{Conclusions}

In summary, PVDF/MXene composites have been achieved by a simple solution blending and compression molding method. The complex structure of MXene leads to an obvious improvement in the thermal properties of PVDF composites. When the loading is increased to $5 \mathrm{wt} \%$, the thermal conductivity of the PVDF/MXene composite is increased to $0.363 \mathrm{~W} \mathrm{mK}^{-1}$, an approximate 1-fold enhancement in comparison with that of neat PVDF. In addition, MXene also exhibits a better performance in improving the mechanical properties of PVDF. In particular, for the PVDF composite containing $5 \mathrm{wt} \%$ MXene, the storage modulus is $7501 \mathrm{MPa}$, corresponding to a $64 \%$ enhancement compared with that of PVDF. This study paves the way for novel, fundamental and application studies of MXenes.

\section{Acknowledgements}

The authors are grateful for the financial support by the National Natural Science Foundation of China (51573201 and 51303034), Natural Science Foundation of Ningbo (2014A610111), Public Welfare Project of Zhejiang Province (2016C31026), Science and Technology Major Project of Ningbo
(2016S1002), and International Science and Technology Cooperation Program of Ningbo (2015D10003). We also thank the Chinese Academy of Science for Hundred Talents Program, the Chinese Central Government for Thousand Young Talents Program, The Key Technology of Nuclear Energy (CAS Interdisciplinary Innovation Team, 2014) and 3315 Program of Ningbo.

\section{References}

1 M. Naguib, O. Mashtalir, J. Carle, V. Presser, J. Lu, L. Hultman, Y. Gogotsi and M. W. Barsoum, ACS Nano, 2012, 6, 1322-1331.

2 M. W. Barsoum, MAX Phases: Properties of Machinable Ternary Carbides and Nitrides, 2013.

3 M. Naguib, V. N. Mochalin, M. W. Barsoum and Y. Gogotsi, Adv. Mater., 2014, 26, 992-1005.

4 M. Naguib, J. Halim, J. Lu, K. M. Cook, L. Hultman, Y. Gogotsi and M. W. Barsoum, J. Am. Chem. Soc., 2013, 135, 15966-15969.

5 J. Zhou, X. Zha, F. Y. Chen, Q. Ye, P. Eklund, S. Du and Q. Huang, Angew. Chem., 2016, 128, 5092-5097.

6 M. Ghidiu, M. Naguib, C. Shi, O. Mashtalir, L. M. Pan, B. Zhang, J. Yang, Y. Gogotsi, S. J. Billinge and M. W. Barsoum, Chem. Commun., 2014, 50, 9517-9520.

7 M. Naguib, M. Kurtoglu, V. Presser, J. Lu, J. Niu, M. Heon, L. Hultman, Y. Gogotsi and M. W. Barsoum, Adv. Mater., 2011, 23, 4248-4253.

8 Y. Dall'Agnese, M. R. Lukatskaya, K. M. Cook, P.-L. Taberna, Y. Gogotsi and P. Simon, Electrochem. Commun., 2014, 48, 118-122.

9 M. Ghidiu, M. R. Lukatskaya, M. Q. Zhao, Y. Gogotsi and M. W. Barsoum, Nature, 2014, 516, 78-81.

10 M. R. Lukatskaya, O. Mashtalir, C. E. Ren, Y. Dall'Agnese, P. Rozier, P. L. Taberna, M. Naguib, P. Simon, M. W. Barsoum and Y. Gogotsi, Science, 2013, 341, 1502.

11 X. Xie, S. Chen, W. Ding, Y. Nie and Z. Wei, Chem. Commun., 2013, 49, 10112-10114.

12 Y. Gao, L. Wang, Z. Li, A. Zhou, Q. Hu and X. Cao, Solid State Sci., 2014, 35, 62-65.

13 Q. Peng, J. Guo, Q. Zhang, J. Xiang, B. Liu, A. Zhou, R. Liu and Y. Tian, J. Am. Chem. Soc., 2014, 136, 4113-4116.

14 O. Mashtalir, M. Naguib, V. N. Mochalin, Y. Dall'Agnese, M. Heon, M. W. Barsoum and Y. Gogotsi, Nat. Commun., 2013, 4, 1716.

15 S. Chen, Q. Wu, C. Mishra, J. Kang, H. Zhang, K. Cho, W. Cai, A. A. Balandin and R. S. Ruoff, Nat. Mater., 2012, 11, 203-207.

16 S. Stankovich, D. A. Dikin, G. H. Dommett, K. M. Kohlhaas, E. J. Zimney, E. A. Stach, R. D. Piner, S. T. Nguyen and R. S. Ruoff, Nature, 2006, 442, 282-286.

17 Y. Lin and J. W. Connell, Nanoscale, 2012, 4, 6908-6939.

18 L. M. Veca, M. J. Meziani, W. Wang, X. Wang, F. Lu, P. Zhang, Y. Lin, R. Fee, J. W. Connell and Y.-P. Sun, Adv. Mater., 2009, 21, 2088-2092.

19 C. Zhi, Y. Bando, C. Tang, H. Kuwahara and D. Golberg, Adv. Mater., 2009, 21, 2889-2893. 
20 W.-L. Song, P. Wang, L. Cao, A. Anderson, M. J. Meziani, A. J. Farr and Y.-P. Sun, Angew. Chem., 2012, 124, 6604-6607.

21 G. Gonçalves, P. A. A. P. Marques, A. Barros-Timmons, I. Bdkin, M. K. Singh, N. Emami and J. Grácio, J. Mater. Chem., 2010, 20, 9927.

22 T. Ramanathan, A. A. Abdala, S. Stankovich, D. A. Dikin, M. Herrera-Alonso, R. D. Piner, D. H. Adamson, H. C. Schniepp, X. Chen, R. S. Ruoff, S. T. Nguyen, I. A. Aksay, R. K. Prud'Homme and L. C. Brinson, Nat. Nanotechnol., 2008, 3, 327-331.

23 U. Yorulmaz, A. Ozden, N. K. Perkgoz, F. Ay and C. Sevik, Nanotechnology, 2016, 27(33), 335702.

24 X.-H. Zha, Q. Huang, J. He, H. He, J. Zhai, J. S. Francisco and S. Du, Sci. Rep., 2016, 6, 27971.

25 V. Ng, H. Huang, K. Zhou, P. S. Lee, W. Que, Z. J. Xu and L. B. Kong, J. Mater. Chem. A, 2017, 5, 3039-3068.

26 X.-H. Zha, J. Zhou, Y. Zhou, Q. Huang, J. He, J. S. Francisco, K. Luo and S. Du, Nanoscale, 2016, 8, 6110-6117.

27 X.-H. Zha, J. Yin, Y. Zhou, Q. Huang, K. Luo, J. Lang, J. S. Francisco, J. He and S. Du, J. Phys. Chem. C, 2016, 120, 15082-15088.

28 H. Zhang, G. Yang, X. Zuo, H. Tang, Q. Yang and G. Li, J. Mater. Chem. A, 2016, 4, 12913-12920.

29 M. Boota, B. Anasori, C. Voigt, M.-Q. Zhao, M. W. Barsoum and Y. Gogotsi, Adv. Mater., 2016, 28, 1517-1522.

30 Z. Ling, C. E. Ren, M.-Q. Zhao, J. Yang, J. M. Giammarco, J. Qiu, M. W. Barsoum and Y. Gogotsi, Proc. Natl. Acad. Sci. U. S. A., 2014, 111, 16676-16681.

31 F. Shahzad, M. Alhabeb, C. B. Hatter, B. Anasori, S. M. Hong, C. M. Koo and Y. Gogotsi, Science, 2016, 353, 1137-1140.

32 Q. Ye, P. Xiao, W. Liu, K. Chen, T. Chen, J. Xue, S. Du and Q. Huang, RSC Adv., 2015, 5, 70339-70344.

33 M. Naguib, M. Kurtoglu, V. Presser, J. Lu, J. Niu, M. Heon, L. Hultman, Y. Gogotsi and M. W. Barsoum, Adv. Mater., 2011, 23, 4248-4253.

34 C. Shi, M. Beidaghi, M. Naguib, O. Mashtalir, Y. Gogotsi and S. J. Billinge, Phys. Rev. Lett., 2014, 112, 125501.

35 M. R. Lukatskaya, O. Mashtalir, C. E. Ren, Y. Dall'Agnese, P. Rozier, P. L. Taberna, M. Naguib, P. Simon, M. W. Barsoum and Y. Gogotsi, Science, 2013, 341, 15021505.

36 A. J. Lovinger, Science, 1983, 220, 1115-1121.

37 A. Salimi and A. A. Yousefi, J. Polym. Sci., Part B: Polym. Phys., 2004, 42, 3487-3495.

38 V. Sencadas, R. Gregorio Filho and S. Lanceros-Mendez, J. Non-Cryst. Solids, 2006, 352, 2226-2229.

39 C. Ribeiro, V. Sencadas, J. L. G. Ribelles and S. LancerosMéndez, Soft Mater., 2010, 8, 274-287.
40 P. Martins, C. Caparros, R. Gonçalves, P. M. Martins, M. Benelmekki, G. Botelho and S. Lanceros-Mendez, J. Phys. Chem. C, 2012, 116, 15790-15794.

41 P. Martins, C. M. Costa, M. Benelmekki, G. Botelho and S. Lanceros-Mendez, CrystEngComm, 2012, 14, 2807.

42 P. Martins, A. C. Lopes and S. Lanceros-Mendez, Prog. Polym. Sci., 2014, 39, 683-706.

43 J. E. Spanier, S. Gupta, M. Amer and M. W. Barsoum, Phys. Rev. B: Condens. Matter Mater. Phys., 2005, 71, 012103.

44 C. Zhao, Q. Wang, H. Zhang, S. Passerini and X. Qian, ACS Appl. Mater. Interfaces, 2016, 8, 15661-15667.

45 P. Yan, R. Zhang, J. Jia, C. Wu, A. Zhou, J. Xu and X. Zhang, J. Power Sources, 2015, 284, 38-43.

46 H. Wang, Y. Wu, J. Zhang, G. Li, H. Huang, X. Zhang and Q. Jiang, Mater. Lett., 2015, 160, 537-540.

47 O. Mashtalir, Dissertations \& theses, Gradworks, 2015.

48 O. Wilhelmsson, J. P. Palmquist, E. Lewin, J. Emmerlich, P. Eklund, P. O. Å. Persson, H. Högberg, S. Li, R. Ahuja, O. Eriksson, L. Hultman and U. Jansson, J. Cryst. Growth, 2006, 291, 290-300.

49 S. Myhra, J. A. A. Crossley and M. W. Barsoum, J. Phys. Chem. Solids, 2001, 62, 811-817.

50 J. Yu, P. Jiang, C. Wu, L. Wang and X. Wu, Polym. Compos., 2011, 32, 1483-1491.

51 H. Dong, L. Fan and C. Wong, Proceedings Electronic Components and Technology Conference 2005, IEEE, 2005, vol. 2, pp. 1451-1454.

52 C. Pan, K. Kou, Q. Jia, Y. Zhang, Y. Wang, G. Wu and A. Feng, J. Mater. Sci.: Mater. Electron., 2016, 27, 11909-11916.

53 L. Meyer, S. Jayaram and E. A. Cherney, IEEE Trans. Dielectr. Electr. Insul., 2004, 11, 620-630.

54 D. Yorifuji and S. Ando, J. Mater. Chem., 2011, 21, 4402.

55 W. Zhou, Y. Gong, L. Tu, L. Xu, W. Zhao, J. Cai, Y. Zhang and A. Zhou, J. Alloys Compd., 2017, 693, 1-8.

56 W. Zhou, C. Wang, T. Ai, K. Wu, F. Zhao and H. Gu, Composites, Part A, 2009, 40, 830-836.

57 P. Bonnet, D. Sireude, B. Garnier and O. Chauvet, Appl. Phys. Lett., 2007, 91, 201910.

58 W. Yu, H. Xie and W. Chen, J. Appl. Phys., 2010, 107, 094317. 59 Z. Kuang, Y. Chen, Y. Lu, L. Liu, S. Hu, S. Wen, Y. Mao and L. Zhang, Small, 2015, 11, 1655-1659.

60 Y. J. Noh, H. S. Kim, B.-C. Ku, M.-S. Khil and S. Y. Kim, Adv. Eng. Mater., 2016, 18, 1127-1132.

61 V. Causin, C. Marega, A. Marigo, G. Ferrara and A. Ferraro, Eur. Polym. J., 2006, 42, 3153-3161.

62 S. Ganguli, A. K. Roy and D. P. Anderson, Carbon, 2008, 46, 806-817.

63 M. Fang, K. Wang, H. Lu, Y. Yang and S. Nutt, J. Mater. Chem., 2009, 19, 7098. 